\title{
Stereoselective Synthesis and Dissolution Studies on Enteric Coated Pellets of (S)-Duloxetine Hydrochloride
}

\author{
Raghunandan Venkatram ${ }^{*}$, Vasanth Kumar Pai and Sriharsha Nagaraj \\ Department of Studies in Quality assurance, JSS college of Pharmacy, Mysore, India \\ Email: hurulihalli@yahoo.com
}

\begin{abstract}
The enantioselective hydrogenation of 2-bromo-1-(thiophen-2-yl)ethanone and further elaboration of thr cyclic carbamate derived from $\gamma$-aminoalcohol to provide a facile synthesis of $(S)$-duloxetine, a potent dual inhibitor of serotonin and norepinephrine reuptake, is described. Enteric coated pellets with polymer load $25 \%$ and $30 \%$ failed to provide required acid resistant to the pellets but very insignificant amount of drug was leached from the coated tablets in acid phase with polymer load $35 \%$ and $40 \%$ in the acidic phase whereas almost the whole amount of drug was released in the buffer phase. The results generated in this study showed that proper selection of polymeric materials based on their physicochemical properties as well as polymer load is important in designing delayed release pellets dosage form with acceptable dissolution profile.
\end{abstract}

Keywords: Enantioselective synthesis, Eudragit L30 D 55 Duloxetine hydrochloride, enteric coating pellets, powder layering.

\section{Introduction}

Duloxetine, a medication with effects on both serotonin and noradrenaline transporter molecules, has recently been approved for the treatment of generalized anxiety disorder. Serotonin and norepinephrine neurotransmitters are intimately involved in a number of neurochemical and physiological processes, such as depression and pain disorders. Selective serotonin or norepinephrine reuptake inhibitors are currently an important class of antidepressants, which includes fluoxetine, nisoxetine, tomoxetine, and duloxetine $^{1}$. They have been approved already as racemates, but some of them are since being redeveloped as 'chiral switches' derived from the established racemates ${ }^{2}$. While fluoxetine and nisoxetine are currently available as racemates, $(S)$-duloxetine [(S)- $N$-methyl-3-(1-naphthyloxy)-3-(2-thienyl)-1propanamine] has gained acceptance in the market because it inhibited serotonin reuptake in rat synaptosomes two times more potently than $(R)$-enantiomer ${ }^{3}$. The $(S)$-duloxetine, a dual inhibitor of both serotonin and norepinephrine reuptake, is effective for the treatment of major depressive disorder and is being considered for treatment of stress-related urinary incontinence. Several different approaches have been reviewed for the synthesis of duloxetine as a racemate or an enantiomerically enriched form ${ }^{4}$. However, there are only a few reports on the asymmetric and catalytic synthesis of duloxetine. One of the methods employs an asymmetric reduction of $\beta$-aminoketone or $\alpha$-cyanoketone/ $\beta$-chloroketone with a chirally-modified LAH complex ${ }^{5}$ or an oxazaborolidine-catalyzed borane respectively ${ }^{6}$. The other involves the chemoenzymatic synthesis, for the most part, lipase-mediated resolution of $\beta$-cyano-, $\gamma-$ chloro-, and $\gamma$-azidoalcohols ${ }^{7}$.

Recently, the application of asymmetric-transfer hydrogenation has been extended to enantioselective hydrogenation of unsaturated carbonyl and imine groups ${ }^{8}$. The asymmetric-transfer hydrogenation, rather,

\footnotetext{
* Corresponding author
} 
offers an operational simplicity, since the reaction does not involve molecular hydrogen and is insensitive to air oxidation, and thus is particularly valuable in scale-up syntheses of active pharmaceutical ingredients. In continuation of our earlier efforts towards the preparation of biologically important compounds, particularly possessing a chiral aminoalcohol unit ${ }^{9}$ we herein report an asymmetric-transfer hydrogenation of 2-tosyloxy-1-(2-thiophenyl)ethanone and further elaboration of a cyclic carbamate to access the facile synthesis of duloxetine.

The enteric coat film coat is a special film coat designed to resist gastric fluids and disrupt or dissolve in small intestine. The enteric coat used to protect drug from degrading in stomach or to minimize the gastric distress caused by some drugs. Enteric coated pellets must empty from stomach before absorption begins. The rate of appearance of blood after giving the enteric coated tablets is the function of gastric emptying. The differences in the gastric emptying from one patient to another or in the same patient with different administration found to be large variability in absorption commonly found in this dosage form. The enteric coated tablets approximately require 0.8 to $5 \mathrm{hrs}$ to travel from stomach to duodenum but enteric coated pellets dispersed in the stomach and pass through pyloric sphincter after a mean residence time in the stomach similar to suspension dosage form ${ }^{10}$. The synthesized $(S)$-duloxetine hydrochloride was formulated as enteric tablets and evaluated for various characteristics including dissolution rate. The possibility of increasing the dissolution rate of duloxetine through formulated enteric coated pellets was investigated.

\section{Experimental}

The dissolution of duloxetine hydrochloride enteric coated pellets was studied by dissolution tester (En/veka ,Germany) using USP apparatus 11 (Paddle method). An appropriate amount of duloxetine hydrochloride enteric coated pellets containing $20 \mathrm{mg}$ of duloxetine in total was used in $900 \mathrm{ml}$ of dissolution medium ( $0.1 \mathrm{~N}$ hydrochloric acid) at $370 \mathrm{C}$ and $100 \mathrm{rpm}$ for 2 hours. After 1 hour $25 \mathrm{ml}$ sample was withdrawn from each vessel and replaced with fresh medium so that the volume remain constant. At the end of 2 nd hour $25 \mathrm{ml}$ sample was withdrawn from each vessel. Drug content of the sample solution i.e. the quantity of drug release was determined by high-performance liquid chromatography (HPLC) method. Then by replacing the acid media after $2 " \mathrm{~d}$ hour, $900 \mathrm{ml}$ dissolution media $\left(\mathrm{KH}_{2} \mathrm{PO}_{4}\right.$ buffer, $\mathrm{pH}$ 6.8) was added in each vessel. Then again the machine was operated at a rotation of $100 \mathrm{rpm}$ at $370 \mathrm{C}$ for next 1 hour. After 1 hour $25 \mathrm{ml}$ sample was withdrawn from each vessel. After appropriate dilution, the drug content of the collected samples i.e. the quantity of the drug release was determined by HPLC method. The HPLC system consisted of a pump (Waters, USA), an auto sampler (INaters, USA), and a UV detector (INaters,USA). The reverse-phase column (C18) O(terra, $5 \mathrm{pm}, 4.6 \mathrm{~mm} \times 25 \mathrm{~cm}$, Waters) was used at ambient temperature. The mobile phase consisted of acetonitrile (40\%) and the flow rate was $1 \mathrm{ml} / \mathrm{min}$. The injection volume was $20 \mathrm{pl}$ and the signal was observed at $218 \mathrm{~nm}$.

The reactions were monitored by TLC using silica gel plates and the products were purified by flash column chromatography on silica gel (230-400 mesh). Melting points were measured on an electrothermal apparatus and were uncorrected. NMR spectra were recorded at $300 \mathrm{MHz}$ for $\mathrm{H}$ and 75

$\mathrm{MHz}$ for ${ }^{13} \mathrm{C}$. Mass spectra were recorded on a GC/MS operating system at an ionization potential of $70 \mathrm{eV}$. Optical rotations were measured on a high resolution digital polarimeter. The ee values of the samples were determined by HPLC analysis using Daicel Chiralcel OD-H chiral column.

\section{(S)-Duloxetine (8)}

To a solution of 7 (171 mg, $1 \mathrm{mmol})$ in DMSO $(5 \mathrm{ml})$, were added sodium hydride $(36 \mathrm{mg} 1.5 \mathrm{mmol})$ and then 1-fluoronaphthalene $(190 \mathrm{mg}, 1.3 \mathrm{mmol})$. After stirring for $8 \mathrm{~h}$, the reaction mixture was partitioned with ethyl acetate and water. After an extractive workup, the combined organic layers were 
dried over sodium sulfate and then concentrated in vacuo. The residue was purified by flash chromatography (ammonium hydroxide/methanol/ dichloromethane, $0.1 / 1 / 4)$ to yield $232 \mathrm{mg}(78 \%)$ of $\mathbf{8}$ : H NMR (300 MHz, CDCl ) $\delta 8.37-8.33(\mathrm{~m}, 1 \mathrm{H}), 7.79-7.74(\mathrm{~m}, 1 \mathrm{H}), 7.50-7.44(\mathrm{~m}, 2 \mathrm{H}), 7.39-7.37$ (m, $1 \mathrm{H}), 7.28-7.18(\mathrm{~m}, 2 \mathrm{H}), 7.05-7.04(\mathrm{~m}, 1 \mathrm{H}), 6.93-6.90(\mathrm{~m}, 1 \mathrm{H}), \underset{13}{6.86-6.84}(\mathrm{~m}, 1 \mathrm{H}), 5.78(\mathrm{dd}, 1 \mathrm{H}, J 7.6$ and $5.3 \mathrm{~Hz}$ ), 2.86-2.78 (m, 2H), 2.50-2.39 (m, 4H), 2.27-2.16 (m, 1H); C NMR (75 MHz, CDCl ) $\delta 153.3$, 145.2, 134.5, 127.4, 126.5, 126.2, 126.1, 125.7, 125.2, 124.6, 124.5, 122.1, 120.5, 106.9, 74.7, 48.3, 39.0, 36.5; EIMS (70eV) m/z (rel intensity) $297\left(\mathrm{M}^{+}, 4\right), 187(80), 153(69), 144(100) ;[\alpha]_{\mathrm{D}}^{20}=+110.5$ (c 1.1, $\mathrm{MeOH}) ;$ lit. $^{7 \mathrm{~b}}[\alpha]_{\mathrm{D}}{ }^{30}=+114(c$ 1, MeOH$) ;$ lit. $_{i}^{15 \mathrm{~b}}[\alpha]_{\mathrm{D}}{ }^{20}=+112$ (c 1, MeOH); HPLC analysis: $95 \%$ ee (Chiralcel OD-H, hexane/Pr OH, 85/15, 0.5 ml/min; $\mathrm{t}_{\mathrm{R}}(S) 18 \mathrm{~min}, \mathrm{t}_{\mathrm{R}}(R) 25 \mathrm{~min}$.

\section{Results and Discussion}

The starting material, 2-bromo-1-(thiophen-2-yl)ethanone 1 was prepared by photochemical reaction of n-bromo succinamide in carbon tetra chloride in the presence of catalytic amount of benzoyl peroxide. The catalytic reaction of 1 (substrate/catalyst molar ratio 500) with $\mathrm{Cp} * \mathrm{RhCl}[(S, S)-\mathrm{TsDPEN}]^{11}$ where $\mathrm{Cp}^{*}$ $=$ pentamethylcyclopentadienyl, effectively performed with an azeotropic mixture of formic acid/triethylamine (molar ratio 5/2) in ethyl acetate to produce (S)-2-tosyloxy-1-(2-thiophenyl)ethanol 2, $[\alpha]_{D}^{27}=-31.3\left(c 1.08, \mathrm{CHCl}_{3}\right)$, in $95 \%$ yield with $95 \%$ ee. It should be noted the observed enantioselectivity was similar to this reported in the corresponding $\alpha$-chloroketone ${ }^{12}$ and thus represented a first successful application of $\alpha$-tosyloxy heteroaryl ketone in transfer hydrogenation with high enantioselectivity. The ee value was measured by chiral HPLC analysis using Daicel Chiralcel OD-H column. The racemic alcohol ( \pm )-2 was prepared by sodium borohydride reduction of $\mathbf{1}$ in THF, and used as standard for $e e$ determination.

In turn, most approaches to synthesis of the $N$-methylamine 7 routinely adopted lithium aluminum hydride reduction in refluxing THF of the ethyl carbamate derived from the aminoalcohol 4 with ethyl chloroformate, or mono-demethylation of the reduced Mannich product with 2,2,2-trichloroethyl formate with $\mathrm{Zn}$ in toluene. In order to circumvent these harsh conditions, we supposed that the formation of a cyclic carbamate ${ }^{13}$ would offer a facile route to an introduction of $N$-methyl group into the $\gamma$ aminoalcohol, as shown in Scheme 1. It was ambitioned that the required aminoalcohol $\mathbf{4}$ can be easily prepared from the tosylate $\mathbf{2}$, a versatile chiral building block. Thus, the tosylate $(S)-\mathbf{2}$ was readily converted into the nitrile 3 without loss of chirality, $[\alpha]_{\mathrm{D} 20}=-39.7\left(c 0.45, \mathrm{CHCl}_{3}\right) ;$ lit. $^{[7 \mathrm{bb}]}[\alpha]_{\mathrm{D}}^{30}=-33.5(c$ $1, \mathrm{CHCl}_{3}$ ), by the treatment of sodium cyanide in DMSO. Subsequently, the nitrile $\mathbf{3}$ was reduced with borane-dimethyl sulfide in refluxing THF to give the $\gamma$-aminoalcohol which was directly cyclized using $\mathrm{N}, \mathrm{N}$-carbonyldiimidazole (CDI) in the presence of catalytic amount of DMAP to obtain the corresponding cyclic carbamate 5 in $71 \%$ yield for the two steps. Indeed, this allowed a facile introduction of the $\mathrm{N}$ methyl group, by the treatment of methyl iodide with sodium hydride in THF to give the $N$-methyl oxazinanone 6. Hydrolysis of the oxazinanone $\mathbf{6}$ by refluxing with lithium hydroxide in aqueous methanol afforded the aminoalcohol 7. The final installation was then carried out by nucleophilic aromatic substitution with 1-fluoronaphthalene by means of sodium hydride in DMSO to afford $(S)$-duloxetine 8 in $78 \%$ yield with $95 \% e e^{14}$. 


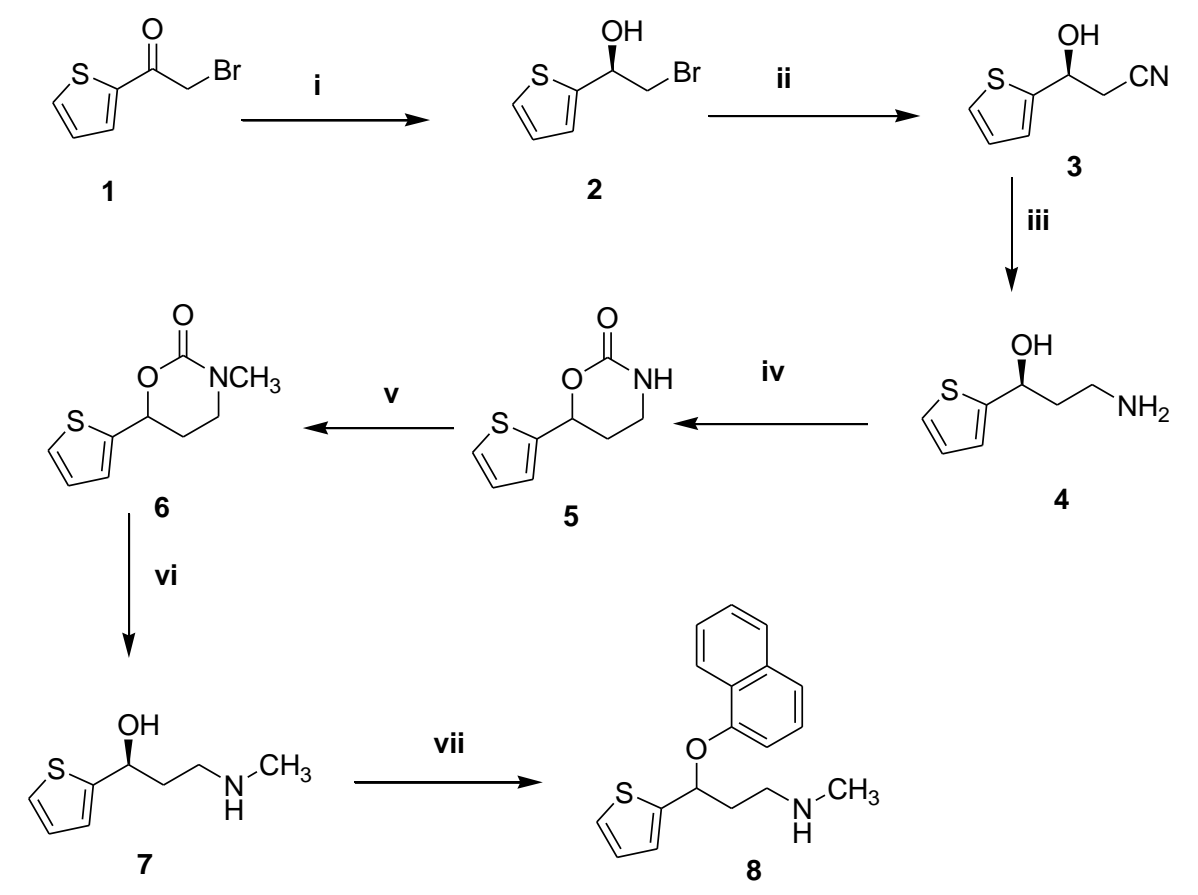

Scheme 1. Asymmetric synthesis of (S)-duloxetine. Reagents and conditions: i) $10 \mathrm{mmol}$ of 1 ( $\mathrm{S} / \mathrm{C}=500), \mathrm{Cp} * \mathrm{RhCl}[(\mathrm{S}, \mathrm{S})-$ TsDPEN], HCO2H/Et3N (molar ratio 5/2, $2 \mathrm{ml}$ ), EtOAc, 3h, 95\%, 95\% ee; ii) NaCN, DMSO, 20h, 88\%; iii) BH3.SMe2, THF, reflux, 2h; iv) CDI, cat. DMAP, CH2Cl2, 8h, $71 \%$ (for 2 steps); v) MeI, NaH, THF, ice-bath, 6h, 89\%; vi) LiOH, MeOH-H2O, reflux, $8 \mathrm{~h}, 84 \%$; vii) 1 -fluoronaphthalene, $\mathrm{NaH}, \mathrm{DMSO}, 8 \mathrm{~h}, 78 \%$.

Duloxetine hydrochloride powder, mannitol and disodium hydrogen phosphate were blended and sieved through 250 micron screen mesh to prepare dusting powder. Disodium hydrogen phosphate and sodium hydroxide were dissolved in purified water. HPMC $5 \mathrm{cps}$ was then dispersed using stirrer to prepare binding solution. NPS (710 micron- $1.0 \mathrm{~mm}$ ) was taken in conventional coating pan and dusting powder was applied on it. The pan rotated at $40 \mathrm{rpm}$ and simultaneously binding solution was sprayed on to the NPS. After completion of drug loading, the nuclei were dried in oven at $100 \mathrm{cc}$ for $5 \mathrm{hrs}$. The dried nuclei was sieved in $1.18 \mathrm{~mm}$ screen mesh followed by $850 \mathrm{~mm}$ screen mesh to get the desired size (850 micron to $1.18 \mathrm{~mm}$ ) and discard under and over sized nuclei. Seal coating suspension was prepared containing HPMC $5 \mathrm{cps}$, PEG-6000, titanium dioxide, sodium hydroxide pellets with the use of silverson stirrer (UK). The dried uncoated nuclei were taken in fluid bed coater and seal coating suspension was sprayed on to it. The sealed coated pellets were dried at $600 \mathrm{C}$ for three hours. Dried seal coated pellets were sieved through $1.18 \mathrm{~mm}$ and 850 micron to get 850 micron to $1.18 \mathrm{~mm}$ seal coated pellets and discard under and oversized nuclei. Enteric coated suspension was prepared by Eudragit L 30 D 55 (ammonio methacrylate copolymer dispersion), talc, triethyl citrate, titanium dioxide and purified water with the use of silversten stirrer (UK). The seal coated pellets were coated using lab coater with Eudragit L 30 D 55 to different thickness equivalent to theoretical polymer load 25\%, 30\%, 35\% and 40\% w/w on dry basis.

The enteric coated pellets were dried in the fluid bed coater at $600 \mathrm{C}$ for 5 hours and then sieved through $1.40 \mathrm{~mm}$ and 850 micron mesh to get 850 micron $1.40 \mathrm{~mm}$ size enteric coated pellets and to discard the under and over sized pellets. In this way all lots of pellets were coated according to the formula for F-1 to F-4 (Table 2). The composition of nuclei, seal pellets are shown in Table 1 Table 2. Machine parameters during fluid coating are shown in Table 3. 
Table 1: Composition of Nuclei and Seal Coated Pellets (Weights are expressed in grams)

\begin{tabular}{|c|c|c|c|}
\hline Nuclei & Quantity & Seal coating & Quantity \\
\hline & & & \\
Duloxetine hydrochloride & 256.24 & Nuclei & 800.00 \\
Disodium hydrogen & 5.53 & HMPC & 50.42 \\
phosphate & 38.30 & PEG-6000 & 6.824 \\
HMPC & 21.56 & Sodium hydroxide pellets & 0.060 \\
Mannitol & 0.452 & Titanium dioxide & 10.48 \\
Sodium hydroxide pellets & 677.90 & Weight of seal coated pellets & 836.00 \\
NPS & 920.00 & Potency of seal coated nuclei & 23.00 \\
Weight of nuclei & 25.30 & & \\
Potency of nuclei & & & \\
\hline
\end{tabular}

Table 2: Codes and Formulation of Duloxetine Enteric Coated Tablets

\begin{tabular}{|c|c|c|c|c|}
\hline Materials & \multicolumn{4}{|c|}{ Formulation codes } \\
\hline & F1 & F2 & F3 & F4 \\
\hline Seal coated pellets & & 200.00 & 200.00 & 200.00 \\
Sodium hydroxide pellets & 0.590 & 0.702 & 0.826 & 0.942 \\
Titanium dioxide & 2.250 & 3.024 & 3.524 & 4.212 \\
Methacrylic acid copolymer dispersion. & 166.67 & 200.00 & 233.23 & 265.65 \\
Purified talc & 1.889 & 2.645 & 2.657 & 3.021 \\
Triethyl dispersion & 8.062 & 9.673 & 11.28 & 12.900 \\
Weight of the enteric coated pellets & 237.00 & 240.00 & 256.34 & 266.43 \\
Potency of enteric coated pellets. & 17.52 & 16.72 & 15.75 & 15.23 \\
\hline
\end{tabular}

Table 3: Machine Parameters during Fluid Bed Coating

\begin{tabular}{|l|l|l|}
\hline \multicolumn{1}{|c|}{ Parameters } & \multicolumn{1}{c|}{ Fluid bed coating } \\
\hline & \multicolumn{1}{|c|}{ Seal coating } & \multicolumn{1}{c|}{ Enteric coating } \\
\hline Batch size & $800 \mathrm{gm}$ & $200 \mathrm{gm}$ \\
Inlet air temperature & $40-45^{\circ} \mathrm{C}$ & $40-45^{\circ} \mathrm{C}$ \\
Outlet air temperature & $30-35^{\circ} \mathrm{C}$ & $30-35^{\circ} \mathrm{C}$ \\
Product temperature & $37-40^{0} \mathrm{C}$ & $37-40^{\circ} \mathrm{C}$ \\
Chamber humidity & $55 \%-60 \%$ & $55 \%-60 \%$ \\
Air flow & $90 \mathrm{~m}^{3} / \mathrm{hr}$ & $90 \mathrm{~m}^{3} / \mathrm{hr}$ \\
Spraying pressure & $1.20 \mathrm{bar}$ & $1.20 \mathrm{bar}$ \\
Spraying rate & $2.0 \mathrm{~g} /$ minute & $3.0 \mathrm{~g} / \mathrm{minute}$ \\
Secondary drying & $60^{\circ} \mathrm{C} / 180$ mins. & $60^{\circ} \mathrm{C} / 300 \mathrm{mins}$. \\
\hline
\end{tabular}


J. Nepal Chem. Soc., vol. 28, 2011

\section{Conclusion}

The $(S)$-duloxetine hydrochloride was successfully synthesized using enantioselective hydrogenation of 2-bromo-1-(thiophen-2-yl)ethanone and further elaboration of through cyclic carbamate derived from $\gamma$-aminoalcohol. Duloxetine hydrochloride loaded pellets were prepared by powder-layering technology. Acid resistant coating with acrylic polymer was done using fluid bed coater at different coating loads and the in vitro release of drug was investigated. The release of drug was found to be a function of polymer load. The results indicated that it is possible to prevent the release of drug in the upper GI tract where the environment is acidic and release the drug in the intestinal region, by developing of multi particulate system coated with suitable $\mathrm{pH}$ dependent polymer.

\section{Acknowledgements}

The authors thank department of studies in industrial chemistry for the laboratory support.

\section{References}

1. Z. Chen, P.Skolnick. Expert Opin. Investig. Drugs., 2007, 16, 1365.

2. I. Agranat, H.Caner, I. J.Caldwel. Nature Rev. Drug Discov., 2002, 1, 753.

3. F.P. Bymaster, E.E. Beedle, J. Findlay, P.T. Gallagher, J.H. Krushinski, S. Bioorg. Med. Chem. Lett., 2003, 13, 4477.

4. L.A. Sorbera, R.M. Castaner, J.Castaner. Drugs Future., 2000, 25, 907.

5. J. Deeter, J. Frazier, G. Staten, M. Staszak, L. Weigel. Tetrahedron Lett., 1990, 31, 7101.

6. W.J. Wheeler, F. Kuo, J.Label. Compd. Radiopharm., 1995, 36, 213.

7. H. Liu, B.H. Hoff, T. Anthonsen. Chirality., 2000, 12, 26.

8. R. Noyori, S. Hashiguchi. Acc. Chem. Res., 1997, 30, 97.

9. D. Lee, J. C.Lee, N.Jeong, K.I.Lee Tetrahedron Asymmetry., 2007, 18, 2662.

10. M.Gibaldi, Biopharmaceutics and clinical pharmaceutics $4^{\text {th }}$ edition, lee and feddiger, USA. p. 68. 11. K.Mashima, T.Abe, K.Tani.Chem. Lett. 1998, 1199.

12. T.Hamada, TTorii, Izawa, T. Ikariya Tetrahedron., 2000, 7411, 60.

13. DehliJ R, V.Gotor.TetrahedronAsymmetry., 2001, 12, 1485.

14. Martindale the complete drug reference, $32^{\text {nd }}$ edition, 2002 p. 291. 